EPJ manuscript No.

(will be inserted by the editor)

SLAC-PUB-10232

CERN-TH/2003-269

ZU-TH 18/03

hep-ph/0311075

\title{
Non-diagonal flavour observables in B and Collider Physics ${ }^{\star}$
}

\author{
T. Hurtha1 and W. Porod ${ }^{2}$
}

1 CERN Theory Division, CH-1211 Geneva 23, Switzerland, SLAC, Stanford University, Stanford, CA 94309, USA

2 Institute for Theoretical Physics, University of Zurich, CH-8057 Zurich, Switzerland

\begin{abstract}
Until now the focus within the direct search for supersymmetry has mainly been on flavour diagonal observables. Recently lepton flavour violating signals at future electron positron colliders have been studied. There is now an opportunity to analyse the relations between collider observables and lowenergy observables in the hadronic sector. In a first work in this direction, we study flavour violation in the squark decays of the second and third generations taking into account results from $B$ physics, in particular from the rare decay $b \rightarrow s \gamma$. Correlations between various squark decay modes can be used to get more precise information on various flavour violating parameters.
\end{abstract}

PACS. 12.60.Jv - 13.25.Hv - 14.80.Ly

\section{Sources of Flavour Violation}

Within the Minimal Supersymmetric Standard Model (MSSM) there are two new sources of flavour changing neutral currents (FCNC), namely new contributions which are induced through the quark mixing as in the SM and generic supersymmetric contributions through the squark mixing. In contrast to the Standard Model (SM), the structure of the unconstrained MSSM does not explain the suppression of FCNC processes that is observed in experiments; this is the essence of the well-known supersymmetric flavour problem. FCNC processes therefore yield important (indirect) information on the construction of supersymmetric extensions of the SM and can contribute to the question of which mechanism ultimately breaks supersymmetry. The experimental measurements of the rates for these processes, or the upper limits set on them, impose in general a reduction of the size of parameters in the soft supersymmetry-breaking terms [1,2,3.

To understand the sources of flavour violation that may be present in supersymmetric models, in addition to those enclosed in the CKM matrix $K$, one has to consider the contributions to the squark mass matrices

$\mathcal{M}_{f}^{2} \equiv$

$$
\left(\begin{array}{cc}
M_{f, L L}^{2}+F_{f L L}+D_{f L L} & M_{f, L R}^{2}+F_{f L R} \\
\left(M_{f, L R}^{2}\right)^{\dagger}+F_{f R L} & M_{f, R R}^{2}+F_{f R R}+D_{f R R}
\end{array}\right)
$$

* Contribution to the International Europhysics Conference on High Energy Physics EPS03, 17-23 July 2003, Aachen, Germany, presented by W.P.

a Heisenberg Fellow where $f$ stands for up- or down-type squarks. The matrices $M_{u, L L}$ and $M_{d, L L}$ are related by $S U(2)_{L}$ gauge invariance. In the super-CKM basis, where the quark mass matrices are diagonal and the squarks are rotated in parallel to their superpartners, the relation reads as $K^{\dagger} M_{u, L L}^{2} K=$ $M_{d, L L}^{2}=M_{Q}^{2}$. In this basis the F-terms $F_{f L L}, F_{f R L}$, $F_{f R R}$ as well as the $D$-terms $D_{f L L}$ and $D_{f R R}$ are diagonal. All the additional flavour structure of the squark sector is encoded in the soft SUSY breaking terms $M_{Q}^{2}$, $M_{f, R R}^{2}\left(=M_{U}^{2}\right.$ for $f=u$ and $M_{D}^{2}$ for $\left.f=d\right)$ and $M f, L R^{2}$ $\left(=v_{u}\left(A^{u}\right)^{*}\right.$ for $f=u$ and $v_{d}\left(A^{d}\right)^{*}$ for $\left.f=d\right)$. Note, that the $A$-matrices are in general non-hermitian.

These additional flavour structures induce flavour violating couplings to the neutral gauginos and higgsinos in the mass eigenbasis, which give rise to additional contributions to observables in the $K$ and $B$ meson sector. At present, new physics contributions to $s \rightarrow d$ and $b \rightarrow$ $d$ transitions are strongly constrained. In particular, the transitions between first- and second-generation quarks, namely FCNC processes in the $K$ system, are the most formidable tools to shape viable supersymmetric flavour models. As was recently emphasized again 4, most of the phenomena involving $b \rightarrow s$ transitions are still largely unexplored and leave open the possibility of large new physics effects, in spite of the strong bound of the famous $\bar{B} \rightarrow X_{s} \gamma$ decay which still gives the most stringent bounds in this sector. Nevertheless, additional experimental information from the $\bar{B} \rightarrow X_{s} \ell^{+} \ell^{-}$decay at the $B$ factories and new results on the $B_{s}-\bar{B}_{s}$ mixing at the Tevatron might change this situation in the near future. Within the present analysis, we take the present phenomenological 
T. Hurth, W. Porod: Non-diagonal flavour observables in B and Collider Physics

situation into account by setting the off-diagonal elements with an index 1 to 0 . Regarding the $b \rightarrow s$ transitions, we restrict ourselves on the most powerful constraint from the decay $\bar{B} \rightarrow X_{s} \gamma$ only.

Two further remarks are in order: within a phenomenological analysis of the constraints on the flavour violating parameters in supersymmetric models with the most general soft terms in the squark mass matrices, we prefer to use the mass eigenstate formalism, which remains valid (in contrast to the mass insertion approximation) when the intergenerational mixing elements are not small. Moreover, a consistent analysis of the bounds should also include interference effects between the various contributions, namely the interplay between the various sources of flavour violation and the interference effects of SM, gluino, chargino, neutralino and charged Higgs boson contributions. In [5] such an analysis was performed for the example of the rare decay $\bar{B} \rightarrow X_{s} \gamma$; new bounds on simple combinations of elements of the soft part of the squark mass matrices are found to be, in general, one order of magnitude weaker that the bound on the single off-diagonal elements $m_{L R, 23}$ which was derived in previous work 2,1, where any kind of interference effects were neglected.

\section{Squark Decays}

Squarks can decay into quarks of all generations if the most general form the squark mass matrix is considered. The most important decays modes for the example under study are:

$$
\begin{gathered}
\tilde{u}_{i} \rightarrow u_{j} \tilde{\chi}_{k}^{0}, d_{j} \tilde{\chi}_{l}^{+} \\
\tilde{d}_{i} \rightarrow d_{j} \tilde{\chi}_{k}^{0}, u_{j} \tilde{\chi}_{l}^{-}
\end{gathered}
$$

with $i=1, . ., 6, j=1,2,3, k=1, . ., 4$ and $l=1,2$. These decays are controlled by the same mixing matrices as the contributions to $b \rightarrow s \gamma$. As this decay mode restricts the size of some of the elements, the question arises as to which extent flavour violating squark decays are also restricted. We will show below that flavour violating decay modes are hardly constrained by current data.

We will take the so-called Snowmass point SPS\#1a 6] as a specific example, which is characterized by $m_{0}=$ $100 \mathrm{GeV}, m_{1 / 2}=250 \mathrm{GeV}, A_{0}=-100 \mathrm{GeV}, \tan \beta=10$ and $\operatorname{sign}(\mu)=1^{1}$. At the electroweak scale one gets the following data: $M_{2}=192 \mathrm{GeV}, \mu=351 \mathrm{GeV}, m_{H^{+}}=$ $396 \mathrm{GeV}, m_{\tilde{g}}=594 \mathrm{GeV}, m_{\tilde{t}_{1}}=400 \mathrm{GeV}, m_{\tilde{t}_{2}}=590 \mathrm{GeV}$, $m_{\tilde{q}_{R}} \simeq 550 \mathrm{GeV}$, and $m_{\tilde{q}_{L}} \simeq 570 \mathrm{GeV}$. In the following we will concentrate on the mixing between the second and third generations. As a specific example we have added a set of flavour violating parameters given in Table 1] the resulting up-squark masses in $\mathrm{GeV}$ are in ascending order:

1 The SPS points are strictly speaking defined by their lowenergy parameters calculated with ISAJET 7.58. In this letter we recalculate these parameters using SPheno 2.0 [7] to include recent theoretical developments in the calculation of the RGEs and the masses.
Table 1. Flavour violating parameters in $\mathrm{GeV}^{2}$ for our example. The corresponding $\operatorname{BR}\left(\bar{B} \rightarrow X_{s} \gamma\right)$ is $4 \times 10^{-4}$.

\begin{tabular}{|ccc|cccc|}
\hline$M_{Q, 23}^{2}$ & $M_{D, 23}^{2}$ & $M_{U, 23}^{2}$ & $v_{u} A_{23}^{u}$ & $v_{u} A_{32}^{u}$ & $v_{d} A_{23}^{d}$ & $v_{d} A_{32}^{d}$ \\
\hline 47066 & 9399 & 46465 & 23896 & -44763 & 14470 & 15701 \\
\hline
\end{tabular}

$408,510,529,542,558$ and 627 and for the down-squark masses we find: $477,525,527,533,564$ and $590 \mathrm{GeV}$. This point is a random one out of 1000 points fulfilling the $b \rightarrow s \gamma$ constraint. For the calculation of $\operatorname{BR}(b \rightarrow s \gamma)$ we have used the formulas given in ref. [8]. Note, that for this SPS\#1a-inspired point both, the chargino and the gluino loops, are important for the calculation of $\operatorname{BR}\left(\bar{B} \rightarrow X_{s} \gamma\right)$. Therefore, there is an interplay between the flavour structure of the down-type squarks and that of the up-type squarks.

In Tables 2 [3] and 4 we collect the branching ratios of squarks and gluinos that are larger than $1 \%$. In addition we also have: $\operatorname{BR}\left(\tilde{u}_{3} \rightarrow \tilde{u}_{1} Z\right)=2.6 \%, \operatorname{BR}\left(\tilde{u}_{3} \rightarrow\right.$ $\left.\tilde{u}_{1} h^{0}\right)=1.2 \%, \operatorname{BR}\left(\tilde{u}_{6} \rightarrow \tilde{g} c\right)=4 \%, \operatorname{BR}\left(\tilde{u}_{6} \rightarrow \tilde{d}_{1} W\right)=2 \%$, $\operatorname{BR}\left(\tilde{u}_{6} \rightarrow \tilde{u}_{1} h^{0}\right)=4.9 \%$ and $\operatorname{BR}\left(\tilde{u}_{6} \rightarrow \tilde{u}_{2} Z\right)=1.8 \%$. Clearly all considered particles have large flavour changing decay modes $^{2}$.

In 9] squark and gluino decays at the LHC have been considered in detail for the point SPS\#1a. It was shown there that lepton and quark distributions can give relatively precise information on the masses of the involved particles by considering the edge variables $m_{l l q}^{\max }, m_{l l q}^{\min }$, $m_{l q}^{\text {low }}$, and $m_{l q}^{\text {high }}$ [9]. These variables are kinematic variables describing the endpoints of jet and lepton distributions in cascades of two body decays of supersymmetric particles [10]. Beside the assumption that flavour changing decays are strongly suppressed, it has also been assumed in that study that squarks of the first two generations have approximately the same mass, within a few per cent. In our example the masses of the squarks range from $408 \mathrm{GeV}$ up to $627 \mathrm{GeV}$. This feature combined with the large flavour violating decay modes will give rise to additional structures in the lepton and jet distributions. In such a case a refined analysis will be necessary to decide whether this additional structure is caused by background, new particles or flavour changing decay modes. In such a scenario a future $e^{+} e^{-}$linear collider (LC) running at 1 $\mathrm{TeV}$ would be of great advantage, in particular if there is some overlap of the running times between LHC and $\mathrm{LC}$. The reason is that at the $\mathrm{LC}$ precise measurements of charginos, neutralinos and sleptons are possible [11. In addition $\tilde{u}_{1}$ and $\tilde{d}_{1}$ are within the reach of a $1 \mathrm{TeV}$ LC in our example. Feeding back the LC information into the LHC analysis will most likely allow for an optimized exploitation of the LHC data.

In ref. 12] several variables have been proposed for extracting information on stops and sbottoms in gluino decays. One class of these variables considers final states containing $b \tilde{\chi}_{1}^{+}$. In our example, three $u$-type squarks contribute with branching ratios larger than $10 \%$, in contrast

\footnotetext{
2 Strictly speaking one should use the expression 'generation violating decay modes' in this context.
} 
Table 2. Branching ratios (in \%) of $u$-type squarks for the point specified in Table 1

\begin{tabular}{|c|cc|cc|cc|cc|cc|cc|}
\hline & $\tilde{\chi}_{1}^{0} c$ & $\tilde{\chi}_{1}^{0} t$ & $\tilde{\chi}_{2}^{0} c$ & $\tilde{\chi}_{2}^{0} t$ & $\tilde{\chi}_{3}^{0} c$ & $\tilde{\chi}_{3}^{0} t$ & $\tilde{\chi}_{4}^{0} c$ & $\tilde{\chi}_{4}^{0} t$ & $\tilde{\chi}_{1}^{+} s$ & $\tilde{\chi}_{1}^{+} b$ & $\tilde{\chi}_{2}^{+} s$ & $\tilde{\chi}_{2}^{+} b$ \\
\hline$\tilde{u}_{1}$ & 4.7 & 18 & 5.2 & 9.6 & $6 \times 10^{-3}$ & 0 & 0.02 & 0 & 11.3 & 46.4 & $2 \times 10^{-3}$ & 4.7 \\
$\tilde{u}_{2}$ & 19.6 & 1.1 & 0.4 & 17.5 & $2 \times 10^{-2}$ & 0 & $6 \times 10^{-2}$ & 0 & 0.5 & 57.5 & $3 \times 10^{-3}$ & 2.9 \\
$\tilde{u}_{3}$ & 7.3 & 3.7 & 20 & 1.4 & $6 \times 10^{-2}$ & 0 & 0.6 & 0 & 40.3 & 3.1 & 1 & 18.5 \\
$\tilde{u}_{6}$ & 5.7 & 0.4 & 11.1 & 5.3 & $4 \times 10^{-2}$ & 5.7 & 0.6 & 13.2 & 22.9 & 13.1 & 0.6 & 8.0 \\
\hline
\end{tabular}

Table 3. Branching ratios (in \%) of $d$-type squarks for the point specified in Table 1

\begin{tabular}{|c|cc|cc|cc|cc|cc|cc|c|}
\hline & $\tilde{\chi}_{1}^{0} s$ & $\tilde{\chi}_{1}^{0} b$ & $\tilde{\chi}_{2}^{0} s$ & $\tilde{\chi}_{2}^{0} b$ & $\tilde{\chi}_{3}^{0} s$ & $\tilde{\chi}_{3}^{0} b$ & $\tilde{\chi}_{4}^{0} s$ & $\tilde{\chi}_{4}^{0} b$ & $\tilde{\chi}_{1}^{-} b$ & $\tilde{\chi}_{1}^{-} t$ & $\tilde{\chi}_{2}^{-} b$ & $\tilde{\chi}_{2}^{-} t$ & $\tilde{u}_{1} W^{-}$ \\
\hline$\tilde{d}_{1}$ & 1.2 & 5.7 & 8.4 & 30.6 & $2 \times 10^{-2}$ & 1.5 & 0.2 & 0.9 & 16.6 & 34.1 & 0.6 & 0 & 0 \\
$\tilde{d}_{2}$ & 17.4 & 5.8 & 5.1 & 15.7 & $7 \times 10^{-2}$ & 7.4 & 0.3 & 09.2 & 9.7 & 19.7 & 0.7 & 0 & 8.8 \\
$\tilde{d}_{4}$ & 14.7 & 21.7 & 11.3 & 2.2 & $5 \times 10^{-2}$ & 10.6 & 0.5 & 8.4 & 22.1 & 3.6 & 1.2 & 0 & 3.4 \\
$\tilde{d}_{6}$ & 1.7 & 0.5 & 20.5 & 6.9 & 0.1 & 0.9 & 1.2 & 1.3 & 40.3 & 10.2 & 3.4 & 11.1 & 1.8 \\
\hline
\end{tabular}

to the assumption that only the two stops contribute. As a consequence we expect that additional structures will be present in the corresponding observables. Moreover, we expect also in this case that a combination of LHC and LC will be useful in the exploration of these structures. A more detailed analysis of the relations between $B$ and Collider physics will be presented in [13.

\section{Conclusions}

We have seen, that large flavour changing decays of squarks and gluinos are consistent with current data from the Tevatron and the $B$ factories. These decays will lead to additional structures in the lepton and jet distributions, which are used to determine the edge variables proposed for the LHC. A linear collider with sufficient energy can in principle measure the branching ratios of the lightest upand/or down-type squarks proving the hypothesis of large flavour violation in the squark sector. This information can then be put back in the analysis of the LHC data.

\section{Acknowledgments}

W.P. is supported by the 'Erwin Schrödinger fellowship No. J2272' of the 'Fonds zur Förderung der wissenschaftlichen Forschung' of Austria and partly by the Swiss 'Nationalfonds'.

\section{References}

1. J. S. Hagelin, S. Kelley and T. Tanaka, Nucl. Phys. B 415 (1994) 293.

2. F. Gabbiani, E. Gabrielli, A. Masiero and L. Silvestrini, Nucl. Phys. B 477 (1996) 321 [arXiv hep-ph/9604387.

3. T. Hurth, Rev. Mod. Phys. 75 (2003) 1159 [arXiv hep-ph/0212304.

4. M. Ciuchini, E. Franco, A. Masiero and L. Silvestrini, Phys. Rev. D 67 (2003) 075016 [arXiv hep-ph/0212397.
Table 4. Gluino branching ratios larger than $1 \%$.

\begin{tabular}{|c|c||c|c|}
\hline Final state & BR [\%] & Final state & BR [\%] \\
\hline$\tilde{u}_{1} c$ & 12.9 & $\tilde{d}_{1} s$ & 7.2 \\
$\tilde{u}_{1} t$ & 5.7 & $\tilde{d}_{1} b$ & 19.8 \\
$\tilde{u}_{2} c$ & 0.4 & $\tilde{d}_{2} s$ & 6.1 \\
$\tilde{u}_{2} t$ & 7.6 & $\tilde{d}_{2} b$ & 4.7 \\
$\tilde{u}_{3} c$ & 0.6 & $\tilde{d}_{3} d$ & 10.0 \\
$\tilde{u}_{4} u$ & 5.5 & $\tilde{d}_{4} s$ & 3.5 \\
$\tilde{u}_{5} u$ & 3.0 & $\tilde{d}_{4} b$ & 4.9 \\
& & $\tilde{d}_{5} d$ & 2.1 \\
\hline
\end{tabular}

5. T. Besmer, C. Greub and T. Hurth, Nucl. Phys. B 609 (2001) 359 [arXiv hep-ph/0105292.

6. B. C. Allanach et al., Eur. Phys. J. C 25 (2002) 113, [arXiv hep-ph/0202233].

7. W. Porod, Comput. Phys. Commun. 153 (2003) 275 [arXiv hep-ph/0301101].

8. F. Borzumati, C. Greub, T. Hurth and D. Wyler, Phys. Rev. D 62 (2000) 075005 [arXiv hep-ph/9911245].

9. G. Polesello, talk given at the 14 Feb. 2003 Meeting of the LHC/LC study group at CERN, http://www.ippp.dur.ac.uk/ georg/lhclc/cern0203/gp.ps B. K. Gjelsten, D. Miller, P. Osland and G. Polesello, contribution to 'Physics at TeV colliders', Les Houches 2003.

10. B. C. Allanach, C. G. Lester, M. A. Parker and B. R. Webber, JHEP 0009 (2000) 004 [arXiv hep-ph/0007009.

11. E. Accomando et al. [ECFA/DESY LC Physics Working Group Collaboration], Phys. Rep. 299 (1998) 1 [arXiv hep-ph/9705442]; J. A. Aguilar-Saavedra et al. [ECFA/DESY LC Physics Working Group Collaboration], arXiv hep-ph/0106315

12. J. Hisano, K. Kawagoe and M. M. Nojiri, Phys. Rev. D 68 (2003) 035007 [arXiv hep-ph/0304214].

13. T. Hurth and W. Porod, in preparation. 